\title{
Luminescence Spectroscopy of Rhodamine Homodimer Dications in Vacuo
}

\section{Steen Brøndsted Nielsen*}

Department of Physics and Astronomy, Aarhus University, Aarhus, Denmark

${ }^{*}$ E-mail sbn@phys.au.dk

The photophysics of dyes strongly depends on their microenvironment, either solvent molecules or nearby charges. Also interactions with other dyes affect the spectroscopical properties. For ionic dyes it can be difficult to quantify the interactions due to solvent screening and/or nearby counter ions. To shed light on intrinsic properties, it is advantageous to perform luminescence experiments in the gas phase, which, however, requires specialized apparatus. In Aarhus we have constructed the LUNA setup, LUminescence iNstrument in Aarhus (Figure 1) [1] that allows us to measure fluorescence from larger ions produced by electrospray ionization. We used this setup to study rhodamine monomer cations as well as homodimers where the two dye cations are separated by methylene linkers, $\left(\mathrm{CH}_{2}\right)_{n}$. I will present the results and compare to spectra measured in solution. Indeed, in the gas phase, we measure significant redshifted emission when there is a nearby charge while in solution solvent screening reduces this effect to almost nothing. The impact of nearby charges may have implications for gas-phase Förster Resonance Energy Transfer experiments where the aim is to reveal the structures of biomolecular ions in the gas phase.

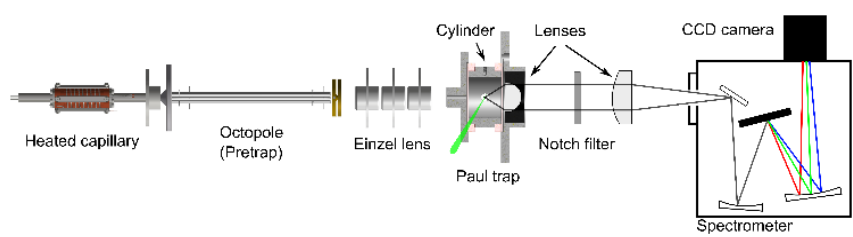

Figure 1. LUNA for gas-phase luminescence experiments on molecular ions.

\section{References.}

[1] M. H. Stockett, J. Houmøller, K. Støchkel, A. Svendsen, S. Brøndsted Nielsen, Rev. Sci. Instrum. 87, 053103 (2016). 